\title{
A review of 117 cases of unprovoked pulmonary embolism and further investigation in patients over 40
}

\author{
Authors: Chloe Angwin, ${ }^{A}$ Alana Rochester ${ }^{A}$ and Muhammad Jawad ${ }^{A}$
}

\section{Introduction}

Venous thromboembolism (VTE) is a common cardiovascular disease with an approximate annual incidence of up to 200 per 100,000 people. ${ }^{1}$ Pulmonary embolism (PE) is a subtype of VTE, and has an estimated incidence of up to 70 per 100,000 people. $^{2}$ A 2018 Cochrane review identified that while investigating for undiagnosed cancers in cases of unprovoked PE may lead to earlier diagnosis of cancers at earlier stages, there is insufficient evidence that detection improves morbidity or mortality. ${ }^{3}$ A 2019 meta-analysis examining rates of cancer in venous thromboembolic disease found a $4.3 \%$ prevalence of occult cancers found in those with $\mathrm{PE},{ }^{4}$ however current National Institute for Health and Care Excellence (NICE) guidance estimates that approximately $20 \%$ of all cases of VTE are due to an active cancer. ${ }^{1}$ This study reviewed cancer rates detected by routine investigation in patients over 40 years old with unprovoked $\mathrm{PE}$, as recommended by current guidance from NICE. ${ }^{1}$

\section{Materials and methods}

This retrospective study examined 117 cases of unprovoked $\mathrm{PE}$ diagnosed in a district general hospital between September 2018 and February 2019. Medical notes were reviewed for initial diagnosis, investigations undertaken, cancer diagnosis and type of cancer.

\section{Results and discussion}

From the cohort of 117 unprovoked PEs, 59\% (n=69) were female and $41 \%(n=48)$ were male. The average age was 69 years \pm 15 . The diagnosis of PE alone was made in $94 \%(n=110)$ patients, while PE with deep vein thrombosis (DVT) was made in $6 \%(n=7)$ patients. Further investigations for underlying cancer as a cause of unprovoked PE were carried out in 77\% $(n=90)$, while $23 \%(n=27)$ did not complete investigations. Underlying cancers were detected after the presentation with an unprovoked $\mathrm{PE}$ in 10 patients (male $\mathrm{n}=4$; female $\mathrm{n}=6$ ), and diagnoses included prostate, lung, breast, renal, gastrointestinal and haematological malignancies.

\section{Conclusion}

This data supports current guidelines to investigate unprovoked PEs for underlying cancers, with a diagnostic rate of approximately $10 \%$. Ideally this should be performed by a dedicated service in order to streamline investigations and onward referrals.

\section{Conflicts of interest}

None declared.

\section{References}

1 National Institute for Health and Care Excellence. Pulmonary embolism. NICE, 2019. https://cks.nice.org.uk/pulmonaryembolism [Accessed 27 October 2019].

2 Bĕlohlávek J, Dytrych V, Linhart A. Pulmonary embolism, part I: Epidemiology, risk factors and risk stratification, pathophysiology, clinical presentation, diagnosis and nonthrombotic pulmonary embolism. Exp Clin Cardiol 2013;8:129-38.

3 Robertson L, Yeoh SE, Broderick C, Stansby G, Agarwal R. Effect of testing for cancer on cancer- or venous thromboembolism (VTE)related mortality and morbidity in people with unprovoked VTE. Cochrane Database Syst Rev 2018;11:CD010837.

4 Jara-Palomares L, van Es N, Praena-Fernandez JM et al. Relationship between type of unprovoked venous thromboembolism and cancer location: An individual patient data meta-analysis. Thromb Res 2019;176:79-84. 